\title{
Urvertrauen zum Leben
}

\section{Wie man die Gesundheit von Kindern und Jugendlichen fördern kann}

Prof. Dr. Heiner Keupp lehrte von 1978 bis zu seiner Pensionierung im Jahre 2008 Gemeinde- und Sozialpsychologie an der Ludwig-Maximilians-Universität München. Er war Vorsitzender der Kommission für den 13. Kinder- und Jugendbericht der Bundesregierung zum Thema »Gesundheitsförderung und Prävention durch Kinder- und Jugendhilfe «. Der Bericht wurde im Frühjahr 2009 der Öffentlichkeit vorgestellt.

E-Mail keupp@psy.uni-muenchen.de
Das Thema der Gesundheitsförderung darf insbesondere im Hinblick auf junge Menschen nicht eng gefasst werden. Der Blick muss sich vielmehr darauf richten, welche Ressourcen Kinder, Jugendliche und ibre Familien benötigen, damit Menschen gesund aufwachsen können.

Professionen der Sozialen Arbeit sind mit ihrem Auftrag in aller Regel auf Personengruppen fokussiert, die Probleme haben, ihr Leben in die eigene Hand zu nehmen. Das liefert uns eine Existenzberechtigung und Arbeit genug. Dabei sollten wir aber tunlichst vermeiden, die fachliche Orchestrierung der Untergangsgesänge zu übernehmen, die seit einigen Jahren zu hören sind.

Es gibt gute Gründe, genau hinzuschauen und $\mathrm{zu}$ differenzieren. Martin Spiewak hat in der Wochenzeitung »Die Zeit « (vom 1. Oktober 2008) vor einer Panikmache in Bezug auf die heranwachsende Generation gewarnt. Er kommt auf der Grundlage belastbarer Daten zu folgenden Aussagen:

- Kinder von heute leben gesünder.

- Kinder sind schlauer als früher.

- Kinder leben heute sicherer.

- Die Kleinfamilie ist nicht totzureden.

- Eltern erziehen - aber anders.

- die Renaissance der Jugend-Tugend Spiewak kommt zu der Schlussfolgerung: "Zu keiner anderen Zeit ging es der Mehrzahl der Kinder in diesem Land so gut wie heute, widmeten sich Eltern so intensiv ihrem Nachwuchs, lebten die Generationen so harmonisch zusammen wie im Jahr 2008. Vergleicht man die Lebensumstände von Familien mit denen von vor zwanzig oder fünfzig Jahren, so hat sich enorm viel verbessert. «

Das mag man als den Teil I der Wahrheit ansehen, den Teil II vermag der Autor durchaus auch zu sehen und zu benennen: »Eine Gruppe profitiert kaum von den Fortschritten bei Bildung und Gesundheit, dem Zugewinn an Sicherheit und Lebenschancen - die Kinder am un- teren Rand der Gesellschaft, die Familien, in denen sich Armut, Arbeitslosigkeit und Vernachlässigung ballen. Dort gibt es tatsächlich Neuntklässler, die laut Pisa-Test gerade einmal auf Grundschulniveau lesen und rechnen können; Jugendliche, die morgens nicht aus dem Bett zur Schule kommen, weil der arbeitslose Vater bis mittags schläft; Migrantenkinder, deren Eltern versuchen, ihre bröckelnde Autorität mit Schlägen wiederherzustellen. Ein Viertel bis ein Fünftel aller Kinder gehört zu dieser Risikogruppe, bei denen die herkömmlichen Instrumente von Schule und Sozialarbeit immer häufiger versagen. «

Die »Studie zur Gesundheit von Kindern und Jugendlichen in Deutschland « (KiGGS) des Robert Koch Instituts gibt dieser salopp formulierten Einschätzung eine solide empirische Fundierung. Die umfangreiche Studie konnte zeigen, dass Kinder aus sozial benachteiligten Familien - häufiger von Übergewicht und Adipositas betroffen sind

- häufiger zu den Rauchenden gehören

- weniger gestillt wurden

- seltener regelmäßig Sport treiben

- mehr Freizeit vor dem Bildschirm verbringen

- sich seltener die Zähne putzen

- häufiger Verhaltensauffälligkeiten zeigen

- häufiger psychische Probleme und Essstörungen haben

- in einem ungünstigeren Familienklima aufwachsen

- geringere personale, soziale und familiäre Ressourcen besitzen

- eine höhere Gewaltbelastung aufweisen

- die Kinderfrüherkennungsuntersuchungen seltener wahrnehmen

- häufiger Aufmerksamkeitsdefizit/Hyperaktivitätsstörungen (ADHS) haben

- häufiger Mütter haben, die während der Schwangerschaft geraucht haben Diesen Wahrheiten ist Rechnung zu tragen, allen katastrophischen Deutungen ist eine empiriegestützte nüchterne Bilanz aufzumachen. 
Und die zeigt uns erst einmal, dass etwa 80 Prozent der Heranwachsenden gut ins Leben finden. Haben wir eigentlich genug Wissen darüber, wie sie das schaffen? Aber diese Frage wird kaum gestellt, vielmehr wird ein sorgenvoller Blick auf die Welt und die Lebenswelt von Heranwachsenden vor allem von einer soziokulturellen Grundstimmung gespeist: Wir sind mit einer erkennbaren allgemeinen großen Verunsicherung konfrontiert, die sich durch Gesellschaft und Politik zieht, und sie stimuliert gegenwärtig nicht die Suche nach vorwärts gerichteten Lösungen oder gar motivierende Utopien, sondern aktiviert die Sicherheitsbedürfnisse.

Eine wohlfahrtsstaatlich orientierte Reformgesellschaft hat sich in eine "Sicherheitsgesellschaft « verwandelt, von der auch die Soziale Arbeit betroffen ist, vor allem wenn es um die Frage geht, welchen Beitrag die Jugendhilfe leisten könnte, um die Vernachlässigung, den Missbrauch oder gar die Tötung von Kindern zu verhindern. Die Frage will ich auf keinen Fall bagatellisieren, aber sie überlagert im Augenblick alle Suchbewegungen, die sich um Lösungen für eine nachhaltige Förderung von Lebenskompetenzen Heranwachsender bemühen.

Unstrittig dürfte sein, dass das Hineinwachsen in diese unruhige und unberechenbare globalisierte Netzwerkgesellschaft zahlreiche Kompetenzen und Ressourcen erfordert, die nicht einfach als »soziales Erbe « von der Elterngeneration an ihre Kinder weitergegeben werden kann. Man könnte holzschnittartig feststellen, dass wir in einer Gesellschaft leben, die gekennzeichnet ist durch

- tiefgreifende kultureller, politischer und ökonomischer Umbrüche, die durch einen global agierenden digitalen Netzwerkkapitalismus bestimmt werden

- sich ändernde biografische Schnittmuster, die immer weniger aus bislang bestimmenden normalbiografischen Vorstellungen bezogen werden können

- durch Wertewandel, der einerseits neue Lebenskonzepte stützt, der aber zugleich in seiner pluralisierten Form zu einem Verlust unbefragt als gültig angesehener Werte führt und mehr selbst begründete Wertentscheidungen verlangt

- veränderte Geschlechterkonstruktionen, die gleichwohl untergründig wirksame patriarchale Normen und Familienmuster nicht überwunden haben
- die Pluralisierung und Entstandardisierung familiärer Lebensmuster, deren Bestand immer weniger gesichert ist kultur durch Partizipation; Selbstwirksamkeitserfahrungen durch Engagement.

\section{"80 Prozent der Heranwachsenden finden gut ins Leben - warum eigentlich? "}

und von den beteiligten Personen hohe Eigenleistungen in der Beziehungsarbeit verlangt

- die wachsende Ungleichheit im Zugang der Menschen zu materiellem, sozialem und symbolischem Kapital, die gleichzeitig auch zu einer ungleichen Verteilung von Lebenschancen führt

- zunehmende Migration und daraus folgenden Erfahrungen mit kulturellen Differenzen und einem Patchwork der Verknüpfung dieser Differenzen zu neuen Hybriditäten, die aber von spezifischen Bevölkerungsgruppen als Bedrohung erlebt werden

- wachsenden Einfluss der Medien, die nicht nur längst den Status einer zentralen Erziehungs- und Bildungsinstanz haben, sondern auch mit ihrem hohen $\mathrm{Maß}$ an Gewaltpräsentation zumindest die Gewöhnung an Gewalt wesentlich fördern

- hegemonialen Ansprüche, die die Mittel von Krieg und Terror einsetzen, um ihre jeweiligen ideologischen Vorstellungen einer Weltordnung jenseits demokratischer Legitimation durchzusetzen.

\section{Sieben Chancen, die Kinder brauchen}

Welche Ressourcen brauchen junge Menschen, um selbstbestimmt und selbstwirksam ihre eigenen Weg in einer so komplex gewordenen Gesellschaft gehen zu können? Ohne Anspruch auf Vollständigkeit lassen sich die folgenden Verwirklichungschancen nennen: Urvertrauen zum Leben; Dialektik von Bezogenheit und Autonomie; Entwicklung von Lebenskohärenz; Schöpfung sozialer Ressourcen durch Netzwerkbildung; materielles Kapital als Bedingung für Beziehungskapital; demokratische Alltags-

\section{Erste Bedingung: Urvertrauen zum Leben}

Für die Gewinnung von Lebenssouveränität ist lebensgeschichtlich in der Startphase des Lebens ein Gefühl des Vertrauens in die Kontinuität des Lebens eine zentrale Voraussetzung, ich nenne es ein Urvertrauen zum Leben. Es ist begründet in der Erfahrung, dass man gewünscht ist, dass man sich auf die Personen, auf die man existentiell angewiesen ist, ohne Wenn und Aber verlassen kann. Es ist das, was die Bindungsforschung eine sichere Bindung nennt, die auch durch vorübergehende Abwesenheit von Bezugspersonen und durch Konflikte mit ihnen nicht gefährdet.

\section{Zweite Bedingung: Dialektik von Bezogenheit und Autonomie}

Eine Bindung, die nicht das Loslassen ermutigt, ist keine sichere Bindung, deswegen hängt eine gesunde Entwicklung an der Erfahrung der Dialektik von Bezogenheit und Autonomie. Schon der deutsch-amerikanische Psychoanalytiker Erik H. Erikson hat uns aufgezeigt, dass Autonomie nur auf der Grundlage eines gefestigten Urvertrauens zu gewinnen ist. Die US-amerikanische Psychoanalytikerin Jessica Benjamin hat in ihrem wichtigen Buch »Die Fesseln der Liebe« deutlich gemacht, dass sich gerade im Schatten der Restbestände patriarchaler Lebensformen Frauen und Männer in je geschlechtsspezifischer Vereinseitigung dem Pol Bezogenheit oder Autonomie zuordnen und so die notwendige Dialektik zerstören. Dabei kommt das Jammergeheul misslingender Beziehungen heraus: »Du verstehst mich nicht! « 


\section{Dritte Bedingung: Entwicklung von Lebenskohärenz}

Lebenskompetenz braucht einen Vorrat von »Lebenskohärenz «. Aaron Antonovsky hat in seinem salutogenetischen Modell nicht nur die individuelle identitäts- und gesundheitsbezogene Relevanz des »sense of coherence « aufgezeigt, sondern auch Vorarbeiten zu einem Familienkohärenzgefühl hinterlassen. Werte und Lebenssinn stellen Orientierungsmuster für die individuelle Lebensführung dar. Sie definieren Kriterien für wichtige und unwichtige Ziele, sie werten Handlungen und Ereignisse nach gut und böse, erlaubt und verboten. Traditionelle Kulturen lassen sich durch einen hohen Grad verbindlicher und gemeinsam geteilter Wertmaßstäbe charakterisieren. Individuelle Wertentscheidungen haben nur einen geringen Spielraum.

Der gesellschaftliche Weg in die Gegenwart hat zu einer starken Erosion immer schon feststehender Werte und zu einer Wertepluralisierung geführt. Dies kann als Freiheitsgewinn beschrieben werden und hat dazu geführt, dass die Subjekte der Gegenwart als »Kinder der Freiheit « charakterisiert werden. Diese werden meist so dargestellt, als hätten sie das Wertesystem der Moderne endgültig hinter sich gelassen. Es wird als »Wertekorsett « beschrieben, von dem man sich befreit habe und nun könnte sich jede und jeder ihren eigenen Cocktail mit Werten zurecht mixen. Das klingt nach unbegrenzten Chancen der Selbstbestimmung und Selbstverwirklichung. Aber diese Situation beschreibt keine frei wählbare Kür, sondern sie stellt eine Pflicht dar und diese zu erfüllen, erfordert Fähigkeiten und Kompetenzen, über die längst nicht alle Menschen in der reflexiven Moderne verfügen.

\section{Vierte Bedingung: Schöpfung sozialer Ressourcen durch Netzwerkbildung}

Wenn wir die sozialen Baumeisterinnen und Baumeister unserer eigenen sozialen Lebenswelten und Netze sind, dann ist eine spezifische Beziehungs- und Verknüpfungsfähigkeit erforderlich, nennen wir sie soziale Ressourcen. Der Bestand immer schon vorhandener sozialer Bezüge wird geringer und der Teil unseres so- zialen Beziehungsnetzes, den wir uns selbst schaffen und den wir durch Eigenaktivität aufrechterhalten (müssen), wird größer. Nun zeigen die entsprechenden Studien, dass das moderne Subjekt keineswegs ein Einsiedler geworden ist, sondern im Durchschnitt ein größeres Netz eigeninitiierter sozialer Beziehungen aufweist, als es seine Vorläufergenerationen hatten: Freundeskreise, Interessengemeinschaften, Nachbarschaftsaktivitäten, Vereine, Selbsthilfegruppen, Initiativen.

Es zeigt sich nur zunehmend auch, dass sozioökonomisch unterprivilegierte und gesellschaftlich marginalisierte Gruppen offensichtlich besondere Defizite aufweisen bei dieser gesellschaftlich zunehmend geforderten eigeninitiativen Beziehungsarbeit. Die sozialen Netzwerke von Arbeiterinnen und Arbeiter beispielsweise sind in den Nachkriegsjahrzehnten immer kleiner geworden. Von den engmaschigen und solidarischen Netzwerken der Arbeiterfamilien, wie sie noch in den 1950er Jahren in einer Reihe klassischer Studien aufgezeigt wurden und in der Studentenbewegung teilweise romantisch überhöht wurden, ist nicht mehr viel übrig geblieben. Das »Eremitenklima « ist am ehesten hier zur Realität geworden.

Unser »soziales Kapital «, die sozialen Ressourcen, sind ganz offensichtlich wesentlich mitbestimmt von unserem Zugang zu ökonomischem Kapital. Für offene, experimentelle, auf Autonomie zielende Identitätsentwürfe ist die Frage nach sozialen Beziehungsnetzen von allergrößter Bedeutung, in denen Menschen dazu ermutigt werden, also sie brauchen »Kontexte sozialer Anerkennung «. Da gerade Menschen aus sozial benachteiligten Schichten nicht nur besonders viele Belastungen zu verarbeiten haben und die dafür erforderlichen Unterstützungsressourcen in ihren Lebenswelten eher unterentwickelt sind, halte ich die gezielte professionelle und sozialstaatliche Förderung der Netzwerkbildung bei diesen Bevölkerungsgruppen für besonders relevant.

\section{Fünfte Bedingung: materielles Kapital als Bedingung für Beziehungskapital}

Ein offenes Identitätsprojekt, in dem neue Lebensformen erprobt und eigener Lebenssinn entwickelt werden, bedarf materieller Ressourcen. Hier liegt das zentrale und höchst aktuelle sozial- und gesellschaftspolitische Problem. Eine Gesellschaft, die sich ideologisch, politisch und ökonomisch fast ausschließlich auf die Regulationskraft des Marktes verlässt, vertieft die gesellschaftliche Spaltung und führt auch zu einer wachsenden Ungleichheit der Chancen an Lebensgestaltung. Hier holt uns immer wieder die klassische soziale Frage ein.

Die Fähigkeit zu und die Erprobung von Projekten der Selbstorganisation sind ohne ausreichende materielle Absicherung nicht möglich. Ohne Teilhabe am gesellschaftlichen Lebensprozess in Form von sinnvoller Tätigkeit und angemessener Bezahlung wird Identitätsbildung zu einem zynischen Schwebezustand, den auch ein "postmodernes Credo « nicht zu einem Reich der Freiheit aufwerten kann.

Dieser Punkt ist von besonderer sozialpolitischer Bedeutung. In allen Wohlfahrtsstaaten beginnen starke Kräfte, die konsensuellen Grundlagen der Prinzipien der Solidargemeinschaft zu demontieren. Das spricht der polnisch-britische Soziologe und Philosoph Zygmunt Bauman in seiner Analyse an: »Der Sozialstaat war darauf ausgerichtet, eine Schicksalsgemeinschaft dadurch zu institutionalisieren, dass seine Regeln für jeden Beteiligten (jeden Bürger) gleichermaßen gelten sollten, so dass die Bedürftigkeit des einen verrechnet würde mit dem Gewinn des anderen.

Wie Bauman aufzeigt, gefährdet gegenwärtig der universalisierte Kapitalismus und seine ökonomische Logik pur das Solidarprinzip: "War der Aufbau des Sozialstaates der Versuch, im Dienste der moralischen Verantwortung ökonomisches Interesse zu mobilisieren, so decouvriert die Demontage des Sozialstaates das ökonomische Interesse als Instrument zur Befreiung des politischen Kalküls von moralischen Zwängen. « (ebd.).

Dramatische Worte wählt Bauman für das erkennbare Resultat dieses Paradigmenwechsels: »Die gnadenlose Pulverisierung der kollektiven Solidarität durch Verbannung kommunaler Leistungen hinter die Grenzen des politischen Prozesses, die massive Freigabe der Preisbindung bei lebenswichtigen Gütern und die politisch geförderte Institutionalisierung individueller Egoismen zum letzten Bollwerk sozialer Rationalität zu haben, ..., (hat) ein veritables `soziales München bewirkt. « (1993). 
Die intensive Suche nach zukunftsfähigen Modellen materieller Grundsicherung ist von höchster Wertepriorität. Die Koppelung sozialstaatlicher Leistungen an die Erwerbsarbeit erfüllt dieses Kriterium immer weniger.

\section{Sechste Bedingung: demokratische Alltagskultur durch Partizipation}

Nicht mehr die Bereitschaft zur Übernahme von fertigen Paketen des »richtigen Lebens", sondern die Fähigkeit zum Aushandeln ist notwendig: Wenn es in unserer Alltagswelt keine unverrückbaren allgemein akzeptierten Normen mehr gibt, außer einigen Grundwerten, wenn wir keinen Knigge mehr haben, der uns für alle wichtigen Lebenslagen das angemessene Verhalten vorgeben kann, dann müssen wir die Regeln, Normen, Ziele und Wege beständig neu aushandeln. Das kann nicht in Gestalt von Kommandosystemen erfolgen, sondern erfordert demokratische Willensbildung, verbindliche Teilhabechancen im Alltag, in den Familien, in der Schule, Universität, in der Arbeitswelt und in Initiativ- und Selbsthilfegruppen. Dazu gehört natürlich auch eine gehörige Portion von Konfliktfähigkeit. Die »demokratische Frage « ist durch die Etablierung des Parlamentarismus noch längst nicht abgehakt, sondern muss im Alltag verankert werden.

\section{Siebte Bedingung: Selbstwirk- samkeitserfahrungen durch Engagement}

Verwirklichungschancen hängen eng mit der Idee der Zivilgesellschaft zusammen. Diese lebt von dem Vertrauen der Menschen in ihre Fähigkeiten, im wohlverstandenen Eigeninteresse gemeinsam mit anderen die Lebensbedingungen für alle $\mathrm{zu}$ verbessern. Zivilgesellschaftliche Kompetenz entsteht dadurch, »dass man sich um sich selbst und für andere sorgt, dass man in die Lage versetzt ist, selber Entscheidungen zu fällen und eine Kontrolle über die eigenen Lebensumstände auszuüben sowie dadurch, dass die Gesellschaft, in der man lebt, Bedingungen herstellt, die allen ihren Bürgerinnen und Bürgern dies ermöglichen « (Ottawa Charta 1986).

\section{Frühe Hilfen}

In den letzten Monaten hat sich in der Öffentlichkeit erkennbar ein Wandel vollzogen: Es wird über unsere Kinder und über neue Formen der Kinderbetreuung gesprochen. Bislang hatte man den Eindruck, dass weder die geringe Geburtenrate in Deutschland noch die Lebenssituation der vorhandenen Kinder ein Thema mit politischer Priorität wären. Die heißen ideologischen Debatten um den Wert der Familie haben einer gezielten Förderung von Kindern und ihren Familien nicht gerade geholfen. Vielleicht funktioniert unsere Gesellschaft nicht anders: Da müssen erst dramatische Dinge passieren, wie mit dem kleinen Kevin in Bremen und in den Medien ausagiert werden, damit politischer Handlungsdruck entsteht. Auf einmal ist das » Kindeswohl « in aller Munde.

In meiner Einschätzung gibt es drei prinzipiell unterschiedliche Zugänge, die durchaus ein paar Überschneidungen haben können: Kindeswohl als staatliche Kontrollaufgabe; Kindeswohl durch Risikoprävention; Gesundheitsförderung als Ressourcenförderung.

- Kindeswohl als staatliche Kontrollaufgabe: Gegenwärtig konzentriert sich der öffentliche, politische und ein Teil des fachlichen Diskurses auf die Optimierung der staatlichen Eingriffsmöglichkeiten. Von Pflichtuntersuchungen mit Sanktionsdrohungen, wenn sie nicht wahrgenommen werden, über Frühwarnsysteme bis hin zu effektiveren Kooperationsformen der Jugendhilfe wird ein breites Maßnahmenbündel diskutiert. Es werden Pläne entwickelt, die kommunalen Ressourcen für Jugendhilfemaßnahmen schwerpunktmäßig zur Optimierung des Kontrollsystems zu nutzen und dafür an anderer Stelle Investitionen zu reduzieren. Diese Sicht passt zu einem Umbau staatlichen Handelns, das sich immer mehr von Sicherheitsprinzipien leiten lässt und von einer Weltsicht bestimmt ist, die überall Bedrohungen sieht.

- Kindeswohl durch Risikoprävention: Neben dieser Perspektive der eher repressive Kontrolle von Familien, die man als Entstehungsherd für Devianz betrachtet, gibt es Programme, die aus der Kenntnis spezifischer Entwicklungsrisiken vor allem in der frühen Kindheit gezielte Präventionsstrategien einsetzen. Am konsequentesten ist der Ansatz der Stadt Dormagen. Da wird jedes geborene Kind zum Anlass für einen Besuch einer Sozialarbeiterin. Es geht um ein "niedrigschwelliges « Angebot, das möglichst nah an der Lebenswelt der Familien angesiedelt ist.

- Gesundheitsförderung als Ressourcenförderung: Aber es gibt Alternativen, die ins Zentrum die Ressourcenförderung von Kindern und Familien im Sinne der Salutogenese rücken und die danach fragen, welche Rechte von Kindern auf Gesundheit, Bildung, materielle Grundsicherung, soziale Einbindung und ökologisch notwendige Lebensbedingungen gesichert werden müssen. Die UNO-Kinderrechte müssen verbindlich und nicht nur als $\mathrm{Ab}$ sichtserklärung ernst genommen werden. Bildungsarmut und mangelnde Gesundheit sind in hohem Maße an prekäre Lebensbedingungen geknüpft, die sich unter den Voraussetzungen einer neoliberal geprägten Politik ständig verschärfen. Wir brauchen stattdessen eine Förderung von Kindern und ihren Familien im Sinne materieller Grundsicherung für alle Kinder und einer umfassenden frühen psychosozialen Förderung entsprechend den Prinzipien von Empowerment und den sich bewährenden Strategien von Kindertageszentren oder Early-Excellence-Projekten und den Mehr-Generationenhäusern, die neben der gezielten Förderung kindlicher Ressourcen auch zivilgesellschaftlichen Prozesse in den Stadtvierteln und Regionen entwickeln, die in einer individualisierten Gesellschaft nicht mehr problemlos gegeben sind.

\section{Hilfe ist mehr als Kontrolle}

Der gegenwärtig dominierende Diskurs zu frühen Hilfen und die Praxis früher Hilfen wirft mindestens so viele Probleme auf, wie er zu lösen vorgibt. Wir können gegenwärtig beobachten, dass durchaus legitime Überlegungen und Strategien zur Optimierung des Kinderschutzes das Aufgabenfeld früher Hilfen gefährlich einengen. Die Konzepte bekommen eine defensive Logik: Es soll etwas verhindert werden und es sollen alle Möglichkeiten gebündelt werden, dies zu erreichen.

Das übersteigerte Bedürfnis nach Sicherheit wird aus einem tiefliegenden 
Angstpotenzial gespeist. Die gesellschaftlichen Strukturveränderungen, deren Folgen Ulrich Beck frühzeitig als »Risikogesellschaft « beschrieben hat, haben uns Orientierungsverluste, Deregulierungen und viele Zukunftsängste beschert. Fast zwei Drittel der Bundesbürger hat das Gefühl, die gesellschaftlichen Entwicklungen nicht mehr zu begreifen oder sie gar beeinflussen zu können. Auf dem Hintergrund wird aus der Risikogesellschaft eine "Sicherheitsgesellschaft « (so Tobias Singelnstein und Peer Stolle (2008), die von einem neuen Typus sozialer Kontrolle bestimmt ist. Sie vertraut immer weniger sozialintegrativen Wohlfahrtsstrategien und legt ein Netz ständig erweiterten Überwachungs- oder Monitoringtechnologien über unsere Gesellschaft. Auch in den Diskursen zu den frühen Hilfen zeigt sich die Logik der Sicherheitsgesellschaft, die den Blick auf das gesamte Feld notwendiger Ansatzpunkte für frühe Hilfen verstellen kann und deren fragwürdige Konsequenzen kaum thematisiert. Einige von ihnen möchte ich noch einmal benennen und zuspitzen:

- Alle Diagnose- und Interventionssysteme, die nicht durch das Hilfesuchverhalten betroffener Personen in Aktion treten, gehen von »Normalität « aus. Diese Vorstellungen bleiben in den Diskursen ungenannt, aber sie existieren.
Gehen sie von einer Pluralität möglicher Normalitäten in einer postmodernen Gesellschaft aus oder von der Figur des leistungswilligen und zuverlässigen Subjekts? Werden kulturelle Differenzen berücksichtigt? Wird gar die Resilienzforschung beachtet, die uns ja erstaunliche Biographien trotz teilweise dramatischer Lebensbedingungen aufgezeigt hat.

- Risikokonstellationen müssen diagnostiziert werden, und wenn die Risiken vollständig erfasst werden sollen, bedarf eines lückenlosen Screenings. Hier kann auch das Prinzip Freiwilligkeit nicht mehr gelten. Das allein würde ja schon eine "Zone des Verdachts" erzeugen: Wer sein Kind dem Screening vorenthält, hat sicher etwas zu verbergen.

- Aus der Psychologie wissen wir, dass Diagnostik und Intervention eng miteinander verzahnt sein müssen, eigentlich überhaupt nicht zu trennen sind. Wie aber sollen flächendeckende Interventionen aussehen, wenn nur eine Verdachtsdiagnostik vorliegt und kein Hilfeersuchen der betreffenden Personen oder Familien signalisiert wird. Wird es dann einen Hausbesuch der Sozialarbeiterin in Begleitung eines Sicherheitsbeamten geben?

- Im Zusammenhang mit einer Ver- dachtsdiagnostik steht die Gefahr des »Labeling « - mit Effekten auf der professionellen wie auch auf der Betroffenenseite: Einer Familie oder einem Kind mit der Annahme zu begegnen, dass sie ein »Risiko « darstellen, erzeugt eine spezifische professionelle Grundhaltung. Auch die betroffenen Menschen werden etwas von dieser Grundhaltung spüren.

- Eine besondere Konsequenz der Selektion von »Problemfällen « ist das, was im englischen als »blaming the victim « bezeichnet wird: eine komplexe gesellschaftliche Problemsituation, vor allem die von Armut und mangelnder gesellschaftlicher Teilhabe, wird den Individuen selbst zur Last gelegt. Sie verhalten sich ja fragwürdig und das ist dann auch die Ursache der Probleme.

- Eine Fachdiskussion umkreist zurzeit das Thema »Exklusion und Inklusion «. Vom »abgehängten Prekariat « spricht die Friedrich-Ebert-Stiftung, von den »Ausgegrenzten der Moderne« Zygmunt Bauman (Bauman 2005). Wie wir der soziologischen Auslegung des Exklusionsthemas entnehmen können, entsteht hier eine Konstellation auf neuem Niveau, die dadurch ausgezeichnet ist, dass neben der objektiven Prekaritätsdiagnose eine subjektive Seite beleuchtet wird, die von Bude und

\section{Der 13. Kinder- und Jugendbericht fordert Gesundheitsförderung als fachlichen Standard}

Der »Bericht über die Lebenssituation junger Menschen und die Leistungen der Kinder- und Jugendhilfe in Deutschland « ist die umfangreiche Studie einer von der Bundesregierung beauftragten Expertenkommission, die in jeder Legislaturperiode von der Bundesregierung herausgegeben wird. Ergänzt werden die Berichte durch eine Stellungnahme der Bundesregierung. Jeder dritte Bericht soll einen Überblick über die Gesamtsituation der Jugendhilfe vermitteln ( $\int 84$ SGB VIII).

Im Mittelpunkt des 13. Kinder- und Jugendberichts der Bundesregierung stehen die Angebote und Maßnahmen der Kinder- und Jugendhilfe im Bereich gesundheitsbezogener Prävention und Gesundheitsförderung. Der Bericht konzentriert sich besonders auf die folgenden Arbeitsfelder der Jugendhilfe und auf deren Schnittstellen zu anderen Systemen: - die allgemein ausgerichteten Angebote, wie etwa die Kindertagesbetreuung oder die Jugendarbeit,

- die Praxisfelder vor allem im Bereich der Hilfen zur Erziehung, in denen vorhandene oder drohende gesundheitliche Beeinträchtigungen ein wesentliches Merkmal der Problemkonstellationen darstellen (z. B. im Zusammenhang mit Suchtgefährdung und psychischen Beeinflussungen, an der Schnittstelle zur Kinder- und Jugendpsychiatrie) und
- die Felder der Integration von und der Arbeit mit jungen Menschen mit drohenden Behinderungen.

Der Bericht fordert, Gesundheitsförderung als fachlichen Standard der Jugendhilfe zu begreifen: »Die Förderung der Gesundheit von Kindern und Jugendlichen muss ein Ziel fachlichen Handelns in der Kinder- und Jugendhilfe werden. Gesundheit von Kindern und Jugendlichen darf nicht als Voraussetzung der Praxis der Kinder- und Jugendhilfe verstanden werden; vielmehr muss die Frage im Mittelpunkt stehen, wie in dem jeweiligen Handlungsfeld, ggf. im Zusammenspiel mit anderen Akteuren, altersgerecht die Gesundheit von Kindern und Jugendlichen gefördert und gesundheitliche Belastungen vermieden werden können. Stärker als bisher bedarf es der Berücksichtigung der gesundheitsbezogenen Folgen von sozioökonomischer und -kultureller Benachteiligung und der Entwicklung entsprechend gegensteuernder Angebote in der Kinder- und Jugendhilfe. «

Der 316 Seiten umfassende 13. Kinder- und Jugendbericht steht als Bundestags-Drucksache 16/12860 im Internet kostenlos zum Herunterladen zur Verfügung.

Internet http://dip21.bundestag.de/dip21/btd/16/128/1612860.pdf 
Lantermann (2006) als »Exklusionsempfinden " bezeichnet wird. Diese "gefühlte Exklusion" ist der Nährboden für Demoralisierung und Hoffnungslosigkeit. Ein solches Exklusionsempfinden kann durchaus durch das Erfasstwerden von einem »Frühwarnsystem « gefördert werden.

- Ein letzter Punkt: Die »Sicherheitsgesellschaft « stellt die defensive Variante des Ordnungstraumes der Moderne dar: Diese hatte und hat den Anspruch, alles Unberechenbare, Uneindeutige, Ambivalente, Fremde und Störende zu beseitigen und eine berechenbare und eindeutige Welt geschaffen. Auch wenn dieser Traum dieser Moderne nur noch selten in naiver Emphase vorgetragen wird, es gibt ihn noch und die Sicherheitsgesellschaft lebt davon. Sie will möglichst alle Risiken eliminieren und verstärkt dafür ihre Sicherheitssysteme. Nach diesen Überlegungen ist es keine Überraschung, wenn ich frühe Hilfen als perfektioniertes Kontrollsystem nicht sinnvoll finde. Elisabeth Helming von Deutschen Jugendinstitut hat es auf den Punkt gebracht: »Alte Denk- und Handlungsmuster der Fürsorge scheinen in einer einseitigen Konzentration der Diskussion von Prävention in Form von Screening und Risikoeinschätzung auf: das gefährdete Kind, das vorrangig das Kind von armen Leuten und Außenseitergruppen ist, das Kind als Objekt der Sorge, statt es im Zusammenhang zu sehen mit den Müttern vor allem, aber auch den Vätern und deren Möglichkeiten und Lebensbedingungen. « (2008, S. 2) Es muss nach meiner Auffassung um eine umfassende Förderung von Verwirklichungschancen gehen, die bei den Lebensbedingungen der Eltern ansetzt und vor allem die frühen Entwicklungsphasen beachtet.

Besonders wichtig erscheint mir eine systematische kommunal-regional ausgelegte Vernetzung von Hilfeangeboten und »Leistungserbringern «. Das neue Kinderschutzgesetz von Rheinland-Pfalz hat hier einen wichtigen Schwerpunkt gesetzt. Es geht ihm vor allem um eine verbindliche Integration von Angeboten und Leistungen des Jugendhilfe- und des Gesundheitssystems in verbindlichen lokalen Netzwerken. Diese Vernetzung ist nicht zum Nulltarif zu haben. Das Land fördert ihren Aufbau und ihre Arbeit pauschal mit sieben Euro pro Kind für jedes Kind im Bezirk des jeweiligen Jugendamtes, das das sechste Lebensjahr noch nicht vollendet hat. Hier scheint mir ein Weg beschritten zu werden, der auch von der Kinder- und Jugendberichtskommission deutlich eingefordert werden wird.

Kinderschutz braucht also die Entwicklung tragfähiger Netzwerkstrukturen aus der Verknüpfung von Jugendhilfe und Gesundheitssystem, die ein systematisches Unterstützungsangebot für Risikosituationen entwickeln können und ein zielgenaues Hilfsangebot machen können. Dafür müssen Ressourcen bereitgestellt werden. Die Regie sollte bei den Jugendämtern liegen, aber sie brauchen dann auch Finanztöpfe, aus denen beispielsweise niedergelassene Ärzte für die investierte Zeit angemessen honoriert werden können.

\section{Sieben Bedingungen für einen guten Start ins Leben}

In ihrer gemeinsamen Stellungnahme »Gesundes Aufwachsen von Kindern und Jugendlichen - Kooperation von Gesundheitswesen und Kinder- und Jugendhilfe « haben die Arbeitsgemeinschaft für Kinder- und Jugendhilfe und der Berufsverband der Kinder- und Jugendärzte festgestellt: »Der beste Weg, gefährdete Kinder vor einer Vernachlässigung und Gefährdung zu schützen, ist der einer Früherkennung und frühzeitigen Hilfe, die ansetzt, bevor sich ungünstige Entwikklungsverläufe stabilisiert haben, und die insbesondere den Abbau von Belastungsfaktoren in den betroffenen Familien vorsieht.«(AGJ, 2006)

In diesem Sinne müssen frühe Hilfen vor allem durch ein Unterstützungsangebot für Eltern von der Schwangerschaft, über die Geburt bis zu den ersten Lebensmonaten und -jahren organisiert werden. Die vielfältigen Modellansätze der Familienbildung sind möglichst flächendeckend anzubieten und das darin enthaltene Prinzip der Selbsthilfe scheint besonders geeignet auch Familien mit Migrationshintergrund und Prekariatserfahrungen zu erreichen.

Familienhebammen sind hier ein mögliches Angebot, aber nicht der Königsweg, weil sie in aller Regel keine umfassende Qualifizierung erfahren haben. Am besten geeignet scheinen da Early-excellence-Projekte, Kinder-Tages-Zentren, Häuser für Familien, Mütter- und Fami- lienzentren bis hin zu Mehr-Generationen-Häusern, die sozialraumbezogen ausgerichtet sind und ein komplexes Angebot machen können. Sie dürfen nicht unter ein Kontrollperspektive wahrgenommen werden, sondern als abrufbare Assistenz und als Orte, an denen sich Familien treffen und austauschen können und damit auch Selbstorganisationswünsche der Betroffenen erreichen.

Nach der wissenschaftlichen Begleitung eines Kinder-Tags-Zentrums (KiTZ) in München-Ramersdorf, einem problembelasteten Stadtbezirk, sehe ich hier einen innovativen Ansatz. KiTZ-Projekte versuchen durch eine Ausweitung des Angebots über eine reine Kindertagesbetreuung hinaus zu Nachbarschaftszentren und familienorientierten Begegnungszentren zu werden. Daran ansetzend geht es auch um eine stärkere Integration von benachteiligten Bevölkerungsgruppen und um die Frage, welchen Stellenwert vorschulische Einrichtungen einnehmen können, um möglichst frühzeitig bestimmten Benachteiligungen oder zumindest deren Chronifizierung entgegenzuwirken. Folgende Schlussfolgerungen lassen sich aus diesem Ansatz ziehen:

- Unterstützung gegen Kinderarmut: Armut verbaut Chancen, vergrößert gesundheitliche Defizite und ist einer der häufigsten Gründe für psychisches Leid. Für betroffene Familien wären Anlaufstellen hilfreich, die frühzeitig und mit einem breitgefächerten Angebot beraten, stützen und durch die Möglichkeit der gesicherten Betreuung von Kindern oft erst die Voraussetzung für einen Einstieg in die Arbeitswelt schaffen. Im Kampf gegen die Folgen der Kinderarmut haben Kinder-TagesZentren einen wichtigen Platz. Ihre niedrigschwelligen Angebote erlauben auch jenen, für die Armut ein tabubeladenes Thema ist, den Zugang.

- Zentren der frühen Förderung: Armut ist einer von mehreren Gründen, aber beileibe nicht der einzige, warum das Thema der frühen Förderung eine solche Brisanz gewonnen hat. Auch andere Faktoren führen dazu, dass Kinder von ihren Eltern vernachlässigt, in ihrer Entwicklung nur mangelhaft gefördert oder gar misshandelt werden. Die Fachwelt ist sich einig, dass Kinder, die unter schwierigen Lebensbedingungen aufwachsen, wirksam vor Vernachlässigung nur dann $\mathrm{zu}$ schützen sind, 
wenn frühzeitig einsetzende Hilfe- und Unterstützungsangebote vorhanden sind.

- Ausgleich von Benachteiligung: Übereinstimmendes Ergebnis vieler Studien zur frühkindlichen Entwicklung ist: Je früher der Förderprozess ansetzt, desto nachhaltiger sind die Ergebnisse. Kinder stark machen bedeutet, sie früh zu fördern.

- Integration von Migrantenkindern: Das Erlernen der Sprache, anderer Werte und Regeln gelingt in der spielerischen Kultur der Kindertageseinrichtungen am besten. Auch kann hier zusätzlichem Förderbedarf am besten Rechnung getragen werden. Gerade die intensive Kooperation mit den Institutionen der Frühförderung und anderen Fördereinrichtungen für kleine Kinder sowie die - in dieser Altersphase leichter gelingende - Kooperation mit den Eltern können die Basis für eine Integrationsarbeit legen, die auch aus demografischen Gründen ausgebaut werden sollte.

- Nationaler und internationaler Trend: Es gibt europaweit eine breite Erfah-

\section{Literatur}

Antonovsky, A. (1997). Salutogenese. Zur Entmystifizierung der Gesundheit. Tübingen: dgvt-Verlag.

Bude, H. \& Lantermann, E.-D. (2006). Soziale Exklusion und Exklusionsempfinden. In: Kölner Zeitschrift für Soziologie und Sozialpsychologie, 58, S. 233-252.

Helming, E. (2008). Alles im Griff oder Aufwachsen in gemeinsamer Verantwortung? Paradoxien des Präventionsanspruchs im Bereich Früher Hilfen. Vortrag im Rahmen der Fachtagung »Frühe Hilfen für Eltern und Kinder « in Tutzing am 20.4.2008.

LAG-Standpunkt (2007). Früherkennung von Risikofamilien und frühe Hilfen für Eltern von Säuglingen und Kleinkindern. In: Erziehungsberatung aktuell, 2/2007, S. 2-5.

Singelnstein, T. \& Stolle, P. (2008). Die Sicherheitsgesellschaft: Soziale Kontrolle im 21. Jahrhundert. Wiesbaden: Verlag für Sozialwissenschaften.

Spiewak, M. (2008). Falsche Panik. Die Zeit, 1.10.2008 Nr. 41. rung, die für eine Integration von gemeinwesenorientierten Ansätzen mit Konzepten der Kinderbetreuung spricht. Insbesondere das Konzept des »Early Exellence Centres «, das von Neuseeland über England seinen Siegeszug angetreten hat, macht dies deutlich. Auf der Basis der offenen Grundidee, »die Förderung des Kindes sollte von Anfang an exzellent sein ", wird eine stark kind- und elternzentrierte Angebotsstruktur in eine sozialräumliche Ausrichtung übersetzt. Diese Erkenntnis führt zu einem schrittweisen Ausbau von Eltern-Kind-Zentren in Deutschland.

- Fachliche Herausforderung und Weiterentwicklungspotenzial: Die Verknüpfung verschiedener Angebote zu ElternKind-Zentren hat sich als innovative Praxis aus verschiedenen Hintergründen heraus entwickelt. Mütterzentren, Familienbildungseinrichtungen und Kindertagesbetreuungseinrichtungen haben sich, mit ähnlichen Erkenntnissen zur Bedarfslage von Eltern, von jeweils anderer Seite kommend angenähert. Dabei ist die Herausforderung eine neue Stufe von Vernetzung. Nun geht es nicht nur um Informationsaustausch und, darauf aufbauend, Fallvermittlung. Kern der fachlichen Weiterentwicklung ist die Koproduktion. Sie garantiert neue und passgenauere Angebote. Eine weitere Herausforderung besteht in der oft geforderten fachlichen Weiterentwicklung der Kitafachkräfte. Ein Teil der fachlichen Weiterqualifizierung geschieht durch den koproduktiven Alltag mit den Kooperationspartnern und den Qualifikationen. Der andere Teil bedarf einer Weiterentwicklung der Aus- und Weiterbildung der pädagogischen Fachkräfte.

- Kinder-Tages-Zentren rechnen sich: Dies ist zunächst eine These, die empirisch noch durch keine Nutzen-Kostenrechnung belegt werden kann, jedoch plausibel erscheint. In einem KinderTages-Zentrum vereinen sich mehrere Einrichtungstypen, ohne dass diese alle in ihrer Infrastruktur (Miete, Verwaltung) bezahlt werden müssen. Selbst wenn beispielsweise die Aufgaben einer heilpädagogischen Förderung dazukommen und entsprechend Personal dazugeschaltet wird, ist dies immer noch billiger, als eine neue Gruppe aufzumachen.
Der Bedarf wird steigen. Wir brauchen Kinder-Tages-Zentren dringend vor allem in Quartieren mit besonderem Förderbedarf, wir brauchen sie aber mittelfristig auch als flächendeckendes Angebot. Viele Untersuchungen sprechen dafür, dass der nach wie vor tiefgreifende gesellschaftliche Wandlungsprozess einen wachsenden Unterstützungsbedarf junger Eltern generiert und diese jungen Eltern zudem auch offener als frühere Generationen von Eltern sind, breitgefächerte Unterstützungsangebote, wie sie ein Kinder-Tages-Zentrum bietet, rechtzeitig anzunehmen.

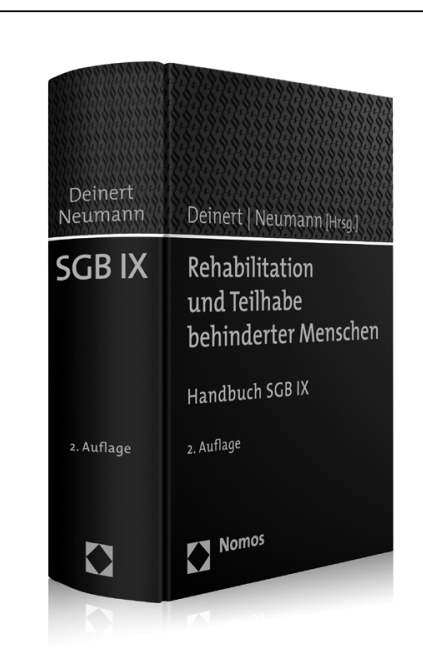

\section{Rehabilitation und Teilhabe behinderter Menschen}

Handbuch SGB IX

Herausgegeben von

Prof. Dr. Olaf Deinert und

Prof. Dr. Volker Neumann

2. Auflage 2009, 789 S., geb., 98,-€, ISBN 978-3-8329-3937-3

Die Neuauflage des Handbuchs SGB IX berücksichtigt u.a. das in Kraft getretene Gesetz zu dem Übereinkommen der UNO über die Rechte von Menschen mit Behinderung, das Gesetz zur Einführung Unterstützter Beschäftigung, die Versorgungsmedizin-VO und das Gesetz zur Förderung der Ausbildung und Beschäftigung schwerbehinderter Menschen.

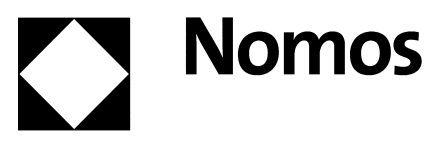

Bitte bestellen Sie im Buchhandel oder versandkostenfrei unter $\downarrow$ www.nomos-shop.de 